\title{
MODELING OF THE LEARNING PROCESS IN ADAPTIVE TRAINING COMPLEXES
}

\author{
Mikhail Krasnyanskiy, Artem Obukhov*, Denis Dedov, Alexandr Siukhin \\ Tambov State Technical University, Russian Federation
}

The article is devoted to the solving of increasing of the effectiveness of training in adaptive training complexes due to individualization of learning process, adaptation for psychological, physical, physiological, anthropometrical and intellectual features of each person. The questions of modeling of learning process in training systems and its study, formalization and software realization are considered. The task of learning process organization in adaptive training complexes (ATC) by optimal way in accordance with selected criteria is stated on the base of developed mathematical model considering the individual features of learners. Using the available approaches to modeling of learning process, we adjusted the psychological, intellectual, physical, physiological and anthropometric metrics concepts, the complexity of the problem is solved. The described statement of a problem of learning process organization, criteria of optimization and mathematical model allow choosing the most suitable forms of studying, methodic, software and hardware tools for learning process on the stage of learning techniques design. The obtained scientific results can be used both at the stage of learning systems design and at the stages of their functioning for the aims of efficiency of education improvement and it's adaptation for the individual features if learners.

Key words: Adaptive training complexes; Qualimetric competence scale; Re-adaptation; Learning

\section{INTRODUCTION}

The contemporary education aims at the individualization of the educational process, adaptation to psychological, physical, physiological, anthropometric and intellectual characteristics of each person in order to improve the effectiveness of the learning process. New methods and forms of learning, both within the framework of professional education and continuous training of professionals throughout the entire working life, are offered [1-3].

One of the most important training tools are training systems that make it possible to acquire with high efficiency the necessary theoretical knowledge and practical skills, as well as accelerate the process of developing professional and universal competences through the use of modern information technologies and visualization tools. In this regard, the adaptive training complexes (ATC) are of particular interest. They include not only software, but also hardware, which can tailor the learning process to specific physical characteristics of an individual. In addition, ATC includes the necessary tools to customize the training course to fill in the gaps in the learners' knowledge or change the complexity of the assignments.

The use of ATC in training and attestation of personnel in manufacturing, transport, medicine and other industries has already shown its effectiveness. However, many aspects in this field require analysis and formalization since many simulators are designed without a systematic study of the theoretical basis. In this article, we will consider the question of modeling of the learning process in ATC, pursuing the main goal of the effectiveness improvement of training using ATC software and hardware. The study and formalization of this process will enable the simplification of the software implementation in ATC and learn- ing process organization in the most appropriate way to meet the chosen criterion.

\section{METHODS}

First, we look at the basic methods and approaches to modeling of the learning process in virtual simulators. The use of ATC does not radically change the entire learning process, but adds some significant features to it. The problem of engineers' training applying modern information technologies was studied in detail in [4]. The authors' approach to the assessment of learner's skills, as well as the systematized psychological metrics plays a big role in modeling of the learning process. This approach works well with ATC and makes it possible to match the psychophysiological characteristics of the trainee and a set of assignments in the learning course, as well as manage the complexity of the assignments.

In [5], the mathematical model of training was considered from the perspective of the main stages of training, the processes taking place in them and a set of rules used for the design of learning materials, assessment and the learning process itself. In terms of ATC, this approach is also quite reasonable because it makes it possible to identify the main stages of the learning process, to apply a set of rules to the development of assignments, and to customize them to the learners' individual needs within ATC operation.

Another example of learning modeling is the context model presented in [6] and obtained on the basis of analyzing knowledge sources and determining the dependence of the growth of the level of competencies from the source of knowledge. 
The approach, outlined in the article, assumes that a person's competences are inseparable from his productivity in the workplace; therefore, diagnostics of workers and professional qualities can directly affect the process and content of training.

Modern theories, concepts, models and examples of training using the latest technologies were described in [7-11]. The authors presented various options for structural and information models of the learning process, studied the main components, but a strict mathematical model was not obtained, because pedagogy is not primarily concerned with this.

Within the development of virtual simulators [12-14], the focus is on modeling of the visualization processes or technical system used in the simulator. The process of learning is evaluated by the data on the learners' progress measured before and after training on the simulators. Such an approach can be considered as simplified assessment. In addition, this approach does not allow implementing adaptive functions of the simulator in terms of knowledge acquisition, level of personal development, considering the individual characteristics.

Thus, we see that there are a sufficient number of approaches to mathematical modelling of learning process, however, within ATC this issue is not sufficiently developed, and the presented mathematical models do not consider an integrated approach to the learning process using modern information systems and technologies, but affect certain aspects. On the other hand, in the field of modelling of training complexes, the main attention is paid directly to the structure of the simulator and the principles of its functioning, while the learning process itself is formalized rather weakly. However, as mentioned above, without a clear formalization of the learning process it is impossible to build it correctly in terms of developing the necessary competencies and optimizing the time and effectiveness of training. Therefore, the actual problem is the development of a mathematical model of the learning process that takes into account individual (i.e., physical, physiological, psychological, intellectual and anthropometric) features of a person, his interaction with ATC, adaptation of the complexity of the tasks performed, which we consider in this article.

We will begin the mathematical modelling of the learning process with its analysis, the separation of input and output data, and consideration of various constraints and control actions.

The main object of the learning process is a set of individual parameters of the trainee, representing a complex of psychological, physical, physiological, anthropometric and intellectual characteristics of an individual to be taken into account when organizing training, especially ATC. The evaluation of these characteristics with the help of a number of metrics allows customizing both the simulator itself and the list of assignments to be performed by the trainee. For example, from the perspective of physical parameters, it can be noted that training of a person with disabilities (problems with sight, hearing, coordination, etc.) requires a varying of exercises depending on physical characteristics, excluding or adding the necessary hardware to enable trainees with physical disabilities to acquire the necessary competences. From the perspective of psychological parameters of an individual, the assessment of personal qualities and intellectual abilities can be used for the selection of assignments to ensure the effectiveness of training [15-17].

The learning process itself can be divided into three main stages:

- preparation (implementation) of the learning process, including the development of virtual simulators, design of learning materials, formulation of assignments, etc.;

- implementation of the learning process in ATC so that the trainees can acquire the necessary competences;

- verification and analysis of the learning outcomes, drawing conclusions about the level of acquisition of competences and overall learning effectiveness.

The competences, developed in the course of the training, directly affect the personality of the trainee, each iteration of the training course allows moving on to increasingly complex assignments, automate their implementation, which in the future will ensure the effective work of trainees in both regular and emergency modes of operation [16].

The effectiveness of the learning process is influenced by the applied training system, i.e. a set of software and hardware, on the basis of which the structural-parametric synthesis of ATC is carried out. On the other hand, the speed of mastering the curriculum depends on the trainee's individual characteristics, which cannot be modified, but which can gradually change during the learning process.

\section{RESULTS}

The learning process can be represented in the form of a structural diagram (Fig. 1).

The diagram shows the cyclical learning process as a single performance of a set of assignments cannot lead to a sufficient level of mastering the necessary competences. Each iteration will take into account both the success of the assignments performed in the previous cycle, and personal development of an individual during the training. Using this approach to the organization of training in ATC, we can achieve significant results in both the speed of learning and its effectiveness.

It is necessary to develop a mathematical model describing the process of training in order to solve the problem of developing an effective methodology of training, ensuring the development of the necessary competences in the given amount considering the economic, legal, and time constraints, as well as individual characteristics of a trainee.

In general, the mathematical model of the learning process can be represented as follows: 


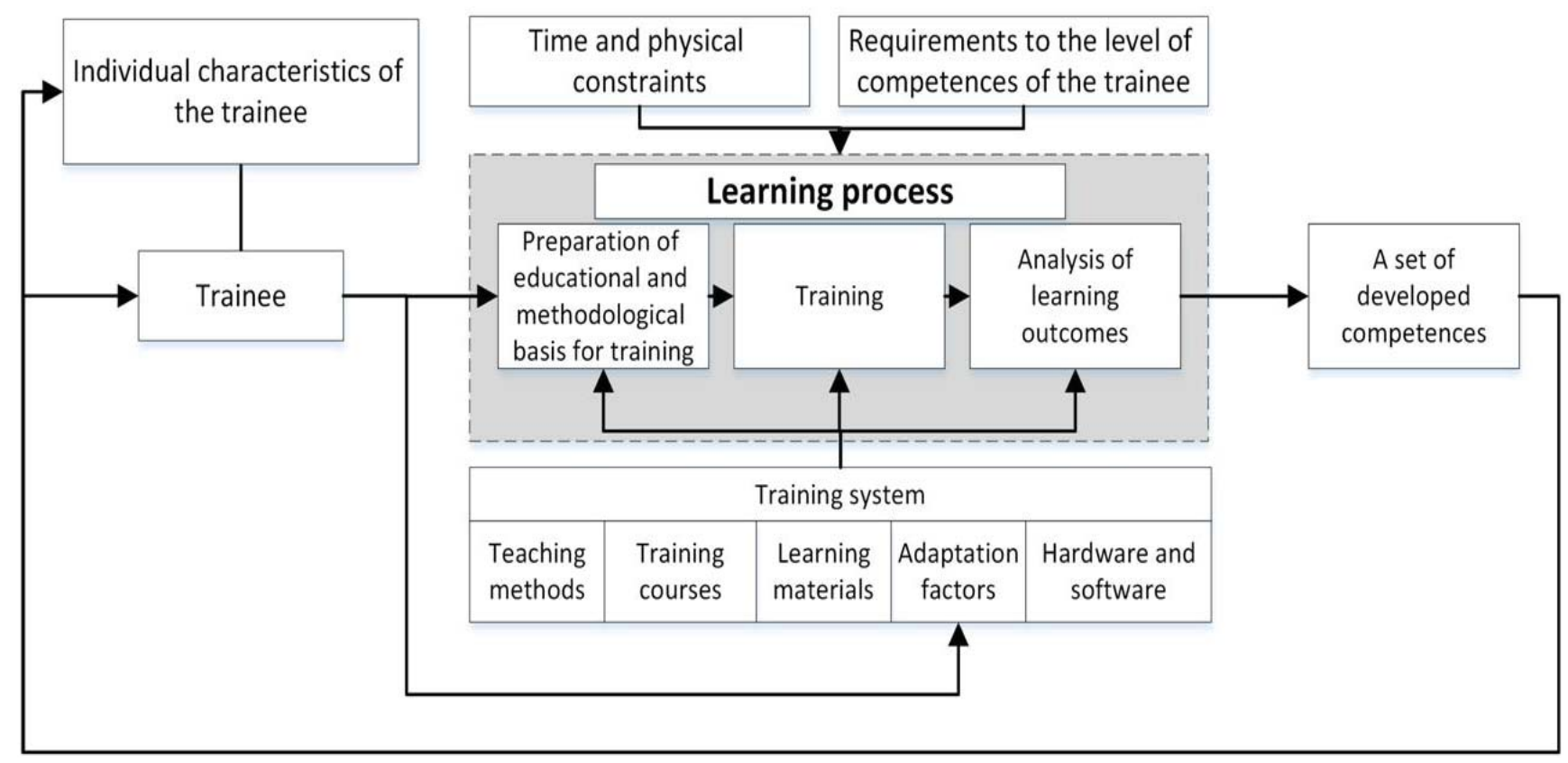

Figure 1: Structural diagram of the learning process

\section{$\operatorname{MTR}(P, T R)=P K$}

The model of the learning process includes 2 input parameters - trainees $P$ and training system TR used to obtain the result (a set of necessary competences $P K$ ). Let us consider each component separately.

So, a set of all users $P$ is follows:

$P=\{P\}$

where each user has the following set of parameters:

$p_{j}-\left(P C_{j}, P M_{j}, P K_{j}\right)$

where $P C_{j}$ - set of individual parameters of a trainee;

$P M_{i}$ - model of the user's activity that can be represented by a set of ordered pairs of production rules $P R_{j}$ and actions $P A_{j}$ performed by the user if the rule is executed:

$P M_{j}=\left\{\left(p r_{j k}, p a_{j k}\right)\right\}$,

$p r_{j k} \in P R_{j}, p a_{j k} \in P A_{j}$

$P K_{j}=\left\{p k_{j k}\right\}-$ set of competences acquired by the user

Set of individual parameters of the trainee, as mentioned above, represents a set of psychological $P S_{i}$, intellectual $P I_{j}$, physical $P H_{j}$, physiological $P L_{j}$ and anthropometrical $P A M_{j}$ parameters:

$P C_{j}=P S_{j} \cup P I_{j} \cup P H_{j} \cup P L_{j} \cup P A M_{j}$.

The elements of a set of psychological characteristics of an individual can be included the following parameters [4]: the level of satisfaction from the process and result of work; the level of satisfaction from material benefits or moral encouragement; the level of introversion/extraversion of an individual; the level of emotional stability of an individual and others. The listed parameters take values from 0 to 1 , depending on the degree of manifestation of this characteristic in the individual's character.
The intellectual parameters $P l_{j}=\left\{p i_{j}\right\}$ of an individual include such characteristics as the level of stochasticity, determinism, logic and imagination in human intelligence; the amount of working memory; the ability to forecast, etc.

The physical characteristics $P H_{j}=\left\{p h_{j}\right\}$ of an individual include speed, strength, reaction speed, endurance, attention, stress resistance, acuity of sight and hearing, etc.

The physiological characteristics $P L_{i}=\left\{p l_{j}\right\}$ of an individual include pulse, the frequency and rhythm of breathing, the volume of sweat and salivation, pressure and others. Anthropometric characteristics $P A M_{j}=\left\{\right.$ pam $\left._{j}\right\}$ of an individual include height, weight, head coverage, limb length, chest volume, distance between eyes, etc.

The evaluation of the effectiveness of trainee's performance can be performed by applying a set of parameters - competence metrics. Each competence corresponds to a set of values of the selected metrics that determine the level of competence acquisition.

The higher is the level, the more complex assignments the learner is able to solve. As an example of such metrics, a five-dimensional qualimetric competence scale (QCS) is used, for example, to assess the quality of training of engineers [4]. We adapt it to the considered mathematical model as follows:

$$
L P: p k_{j k} \rightarrow\left(a_{j k}^{P}, b_{j k}^{P}, c_{j k}^{P}, \operatorname{pol}_{j k}^{P}, \operatorname{chl}_{j k}^{P}\right),
$$

where $L P$ - function that aligns each competence $p k_{j k}$ of the trainee $p_{j}$ with the level of its development in QCS;

$a_{j k}^{P} \in A$ - level of development of formalization abilities by competence $p k_{j k}$ (ability to detect and formalize the problem); 
$b_{j k}^{P} \in B \quad$ - level of development of constructive abilities by competence $p k_{j k}$ (ability to construct the necessary solution to the problem);

$C_{j k}^{P} \in C$ - level of development of executive abilities by competence $p k_{j k}$ (ability to implement a solution);

pol $_{j k}^{P} \in P O L$ - completeness of knowledge of trainee within the competence $p k_{j k}$ (set of facts used in the process of problem solving);

$\operatorname{chl} l_{j k}^{P} \in C H L$ - integrity of the knowledge of trainee within the competence $p k_{j k}$ (set of links and patterns between facts);

$A, B, C$ - set of all formalization, constructive and executive abilities that correspond to the stages of recognition of the problem, the search for its solution and its realization. The values of the elements of sets can be arranged normalized (from 0 to 1 ) or, for example, in specific values of the amount of work performed by the trainee for some time (assignments/ hour).

$P O L, C H L$ - set of values of the completeness and integrity of knowledge, given in percentage to the total volume of theoretical and practical knowledge.

We note that the parameters characterize the trainee's practical skills in solving the problems, and $\mathrm{POL}, \mathrm{CHL}$ constitute a theoretical basis. We show the level of development of the trainee within the competence using QCS:

The dash line defines the area of competence acquisition; the wider this area is, the greater the range of assignments of varying complexity a trainee can solve within the framework of this competence. It is desirable for the area to develop evenly across all five scales to exclude the possibility of producing a "pure" theorist or practitioner.

Thus, we come to the definition of the complexity of the problems solved in the learning process. Within the overall learning process, each assignment $z_{m} \in Z$ has its level of complexity:

$$
D I F F: z \rightarrow\left\{a_{m k}^{Z}, b_{m k}^{Z}, c_{m k}^{Z}, p o l_{m k}^{Z}, c h l_{m k}^{Z} \mid k=1 . . K_{m}^{Z}\right\},
$$

where

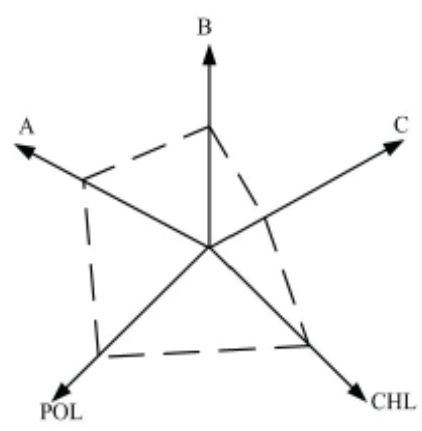

Figure 2: Scale for determining the level of development $L P\left(p_{j}\right)$ of human abilities $a_{m k}^{Z}, b_{m k}^{Z}, c_{m k}^{Z}$, pol $_{m k}^{Z}, c h l_{m k}^{Z}$ - specified levels of complexity of the assignment by the relevant scales within the competence $p k_{j k}$ : formalization, constructive and executive abilities, completeness and integrity of knowledge. Thus, the complexity of the assignment is determined by the complexity of formalization, construction and execution of the solution, as well as the requirements for the completeness and integrity of knowledge for all the necessary $k-m\left(k=1 . . K_{m}^{Z}\right)$ competences. Then the user can successfully solve the task under the following conditions:

$\left\{a_{m}^{Z} \leq a_{j k}^{P}, b_{m}^{Z} \leq b_{j k}^{P}, c_{m}^{Z} \leq c_{j k}^{P}\right.$,
$\left.p o l_{m}^{Z} \leq \operatorname{pol}_{j k}^{P}, \operatorname{chl}_{m}^{Z} \leq c h l_{j k}^{P} \mid k=1 . . K_{m}^{Z}\right\}$.

The possibility of a successful solution of the problem can be represented in the graphical form for one of the competences $p k_{j k}$ as follows:

Thus, by covering the necessary area for solving the problem (the thick line in the figure) with a field of knowledge of a person's competence (dash line), the problem can be solved. Otherwise, it is necessary to increase the insufficient indicators to the required level with the help of various kinds of training tasks, theoretical training, or their combination, which allows achieving the required level of competence in the fastest way.

Considering the complexity of the tasks, it is impossible not to touch upon such a thing as a development zone [5], which allows evaluating the range of tasks that the learner is able to solve at the current level of abilities. Tasks of increased complexity, located outside this zone, allow you to transfer a person from a comfortable state to a stressful situation, activating all of his resources. We

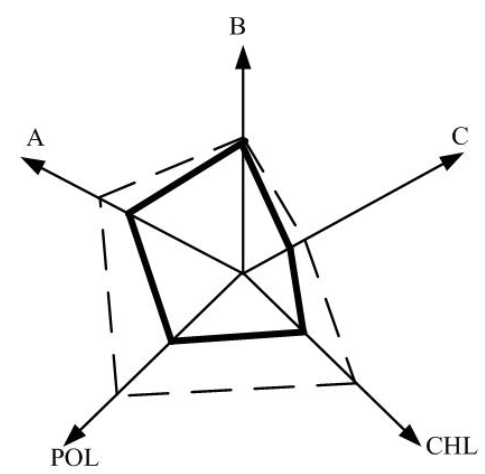

Figure 3: An example of the correlation between the trainee's level of development and complexity of assignment

use the following ratio to determine the zone of development:

$v d_{j} \rightarrow\left\{\left(z_{m}, t z_{m}, r z_{m}\right)\right\}$,

where $v d_{j}$ - development zone of an individual $p_{j}$, which assigns to each trainee information about previously performed tasks $z_{m}$, time of their fulfilment $t_{\mathrm{zm}}$ and evalua- 
tion of the solution correctness $r_{z m}$.

Thus, we can say that the trainee $p$, with the development zone $v d_{j}$ can successfully solve the assignment with a given complexity $\operatorname{DIFF}\left(z_{m}\right)$ if previously he solved successfully the problems with a complexity equal to or greater than $\operatorname{DIFF}\left(z_{m}\right)$. If $\operatorname{DIFF}\left(z_{m}\right)$ exceeds the development zone $v d_{j}$, then the probability of solution is significantly reduced, but if the assignment is successfully completed, it confirms the significant growth of professional and universal competences.

Next, we consider the formalized representation of the learning system within the mathematical model:

$T R=\left(T R_{C}, T R_{I N F}, T R_{R E S}, T R_{A D}, T R_{M}\right)$,

where $T R_{C}$ - set of training courses;

$T R_{I N F}$ - set of reference and learning materials;

$T R_{R E S}$ - set of learning outcomes;

$T R_{A D}$ - set of adaptation factors;

$T R_{M}$ - set of teaching methods.

The learning process is divided into 3 stages:

$F R$ - stage of preparation of the educational and methodological base for training;

$F T$ - stage of training;

$F A$ - stage of analysis of learning outcomes.

We consider each of the stages separately.

$$
\begin{aligned}
& F R=\left(T R_{M}, T R_{I N F}, T R_{A D}\right) \\
& T R_{M}=(\text { TRules }, F O)
\end{aligned}
$$

TRules $=$ TRules $_{A} \cup$ TRules $_{O} \cup$ TRules $_{U}$

where

TRules - set of rules for the formation of teaching methods [5];

$F O=\left\{f o_{j}\right\}-$ forms of training used in the learning process;

TRules $_{A}$ - rules by which the learning tools are formed; TRules $_{O}$ - rules of the learning process;

TRules $_{U}$ - rules forming the control actions.

TRules $_{A}$ include the following categories of rules:

TRules $_{A 1}$ - set of rules of the form "IF < training material $>$ is necessary for the organization of the <training course $>$, THEN it is necessary to implement the <training material> in <a set of reference and training materials>", which are used for the development of tools for training materials $T R_{I N F}$ :

$$
\begin{aligned}
& \text { TRules }_{A 1}: I N F_{T B} \cup I N F_{P B} \cup I N F_{H B} \cup \\
& \cup I N F_{V T} \cup I N F_{D S} \rightarrow T R_{I N F}
\end{aligned}
$$

$I N F_{T B,} I N F_{P B,} I N F_{H B} I N F_{V T,} I N F_{D S}$ - textbooks, workbooks, tutorials, video tutorials, demonstration scenes, and other interactive learning materials. All listed training materials are formed in accordance with a set of rules.

TRules $_{A 2}$ - set of rules of the form "IF for the implemen- tation of <teaching methods> <training system components $>$ are necessary, THEN they are included in <set of hardware and software components of training system>", determining the hardware HW and software SW components of the training system (for example, testing terminals, training equipment, virtual and real simulators, etc.) used in the learning process:

TRules $_{A 2}: \mathrm{TR}_{M} \rightarrow \mathrm{SW}, \mathrm{TR}_{M} \rightarrow \mathrm{HW}$

TRules $_{A 3}$ - set of rules of the form "IF the <assignment $>$ of $<$ training course $>$ is selected, THEN < learning outcome $>$ is evaluated using the <methodology >". They are used to form the learning outcomes of the training process:

TRules $_{A 3}:\left(T R_{C}, T R_{M}\right) \rightarrow T R_{R E S}$.

TRules ${ }_{O}$ regulate the learning process:

TRules $_{01}$ - rules for assessing the individual parameters of the trainee of the form "IF the <trainee> is analyzed, THEN the values of <parameters> correspond to him":

TRules $_{01}: p_{j}-P C_{j}$

TRules $_{\mathrm{O} 2}$ - rules for assessing the level of competences of the trainee of the form "IF the <trainee> is analyzed, THEN the level of <competences> corresponds to him»: TRules $_{\mathrm{O} 2}: p_{j}-P K_{j}$

TRules $_{\mathrm{O} 3}$ - rules of adaptation of the learning process to the individual needs of the trainee of the form "IF the $<$ learning outcomes> are obtained, THEN a set of <adaptation factors $>$ is formed":

TRules $_{O 3}=T R_{\text {RES }}-T R_{A D}$

TRules $_{\mathrm{O} 4}$ - rules for changing the level of competences (transition to a new level of development of the trainee of the form "IF <training course $>$ is completed, THEN the level of <competence metrics> changes":

where $\Delta a_{j k}^{P}, \Delta b_{j k}^{P}, \Delta c_{j k}^{P}, \Delta p o l_{j k}^{P}, \Delta c h l_{j k}^{P}$ - increment of the corresponding competence metrics $p k_{j k}$, the value of which directly depends on the quality of the learning process TR.

TRules $_{05}$ - rules for assessing the knowledge and skills TRules $_{O 4}:\left(p_{j}, T R, a_{j k}^{P}\right) \rightarrow a_{j k}^{P}+\Delta a_{j k}^{P}$,

$\left(p_{j}, T R, b_{j k}^{P}\right) \rightarrow b_{j k}^{P}+\Delta b_{j k}^{P}$,

$\left(p_{j}, T R, c_{j k}^{P}\right) \rightarrow c_{j k}^{P}+\Delta c_{j k}^{P}$,

$\left(p_{j}, T R, p o l_{j k}^{P}\right) \rightarrow \operatorname{pol}_{j k}^{P}+\Delta p o l_{j k}^{P}$,

$\left(p_{j}, T R, c h l_{j k}^{P}\right) \rightarrow c h l_{j k}^{P}+\Delta c h l_{j k}^{P}$,

of the trainee of the form "IF $<$ trainee $>$ completed $<$ training course>, THEN we obtain <learning outcomes>":

TRules $_{\tilde{\mathbb{N} 5}}:\left(p_{j}, T_{R} R_{E}\right) \rightarrow T R \quad$.

TRules $_{06}$ - rules for customizing the individual course for the trainee in accordance with the adaptation factors of the form "IF < adaptation factor>, THEN change <training course>":

TRules $_{\tilde{\tilde{\Omega}} 6}: T R_{A D} \rightarrow T R$. 
TRules $_{U}$ are used to forming the control actions:

TRules $_{U 1}$ - set of rules of the form "IF <control action>, THEN change <training course>", determining the control actions for the process of forming training courses;

TRules $_{U 2}$ - set of rules of the form «IF <control action>, THEN change <methodology > of assessment of <learning outcomes>", determining the control actions for the process of assessing the learning outcomes;

TRules $_{U_{3}}$ - set of rules of the form "IF <control action>, THEN change <assignments $>$ of <training course >", determining the control actions for the process of forming the sequence and complexity of assignments.

The learning process $F T$ is to increase the level of development of competences $P K_{j}$ of the trainee over a period of time $\Delta t_{T R}$ :

$F T:\left(p_{j}, T R\right) \rightarrow\left\{\left(a_{j k}^{P}+\Delta a_{j k}^{P}, b_{j k}^{P}+\Delta b_{j k}^{P}\right.\right.$,

$\left.\left.c_{j k}^{P}+\Delta c_{j k}^{P}, p o l_{j k}^{P}+\Delta p o l_{j k}^{P}, \operatorname{chl}_{j k}^{P}+\Delta c h l_{j k}^{P}\right)\right\}$,

$t_{T R} \rightarrow t_{T R}+\Delta t_{T R}$,

where $P K_{\mathrm{j}}$ - level of competences at the time $t$;

$t_{T R}$ - total training time,

$\Delta t_{T R}$ - time of the current iteration of training.

Unlike the training stage, where the metrics of professional competences increase, the analysis stage directly checks whether the level of competences corresponds to the tasks assigned to the trainee. This verification is carried out by solving a number of problems of a certain complexity:

$F A:\left(p_{j}, T R\right) \rightarrow T R_{R E S}$.

Each task has a set of results, determined by the abilities and knowledge of the trainee at the time. Evaluating the results, one can adjust the training program in accordance with the rules TRules $_{07}$

Thus, we have considered three main stages of the learning process. As noted above, this process is cyclical and repetitive and is aimed at achieving the stated goal in the form of necessary competences with a set level.

The formulation of the problem of optimizing the learning process cannot be reduced to the application of one criterion, since the training system can be subject to different economic (the cost of development and maintenance) and constructive conditions (the use of certain forms of training, hardware and software components of ATC, the requirements for system performance on certain equipment). It is necessary to take into account the number of trainees and economic effectiveness of the training system. The most effective tool is not always justified in terms of total costs. On the other hand, a cheap and simple solution can significantly reduce the quality of training and the time it takes.

Thus, to find the optimal solution from various points of view, we set a multi-criteria formulation of the problem in the following form: it is necessary to form the learning process with the given parameters of the training system $T R$ for trainees in the number $n P$, where the trainees can achieve the maximum level of development of competences $L P\left(p_{i}\right)$ over the minimum training time and with minimal economic costs ER:

$$
\left\{\begin{array}{l}
\sum_{j=1}^{n P} t_{T R}\left(p_{j}, T R\right) \rightarrow \min , \\
E R(T R) \rightarrow \min , \\
\sum_{j=1}^{n P} L P\left(p_{j}\right) \rightarrow \max ,
\end{array}\right.
$$

when performing the relations of the mathematical model (1-24) and restrictions on:

- compliance with mastered competencies $P K$ required by $P K^{*}$ :

$$
P K \subset P K^{*},
$$

- total training time:

$$
\begin{gathered}
\sum_{j=1}^{n P} t_{T R}\left(p_{j}, T R\right) \leq t_{T R}^{*}, \\
\text { - economic resources: }
\end{gathered}
$$

$E R(T R) \leq E R^{*}$,

where $E R$ - total economic costs for the implementation and maintenance of the training system per person;

$E R^{*}$ - maximum possible costs for the implementation and maintenance of the training system per person;

$P K^{*}$ - set of required competences;

$t_{T R}{ }^{*}$ - maximum possible training time.

Training can be conducted in a developing direction with the use of tasks of increased complexity with the aim of increasing the overall labor intensity of the tasks performed and, consequently, increasing the level of human development more effectively. Determining the optimal relationship between quality, training time and its cost is a non-trivial task of multi-criteria optimization. Therefore, it is impossible to say with confidence that the use of expensive and effective learning technologies is always justified; each case requires careful analysis and selection of the optimal solution from various points of view.

\section{CONCLUSION}

The training complexes proved to be effective for personnel training at various spheres of human activity; however, the issue of mathematical modeling of the learning process is not addressed at ATC. The absence of a clear and detailed formalized model of the learning process leads to a paradoxical result: a designed virtual simulator meets all structural requirements, but the effectiveness of learning on it does not cover up the material and time resources invested in the development. In the cited works, the learning process is studied from a pedagogical and methodological point of view without taking into account the specifics of using training complexes. 
The proposed mathematical model of the learning process includes the known parameters (psychological, intellectual, physical, physiological and anthropometric human metrics, complexity of the solved problem, human development zone), adapts them to ATC subject area and allows evaluating the effectiveness of training at the design and operation stage of the training complex.

Within the article, an original formulation of the problem for the learning process optimization is derived, distinguished by the selected criteria (economic costs, quality and learning time), which permits to determine the optimal set of software and hardware of the training complex to provide the maximum efficiency of the learning process.

The formulation of the mathematical model and the optimization problem are a promising direction for the further research on the formalization, analysis and improvement of the effectiveness of the learning process despite the large amount of upcoming experimental studies. Further work will be associated with the analysis of experimental data in solving certain tasks of the learning process organization with the involvement of focus groups.

\section{ACKNOWLEDGEMENTS}

The work was supported by the Russian Ministry of Education as part of the project part (project 8.2906.2017 ( PP) and on the basis of the center for collective use "Digital engineering".

\section{REFERENCES}

1. Peet, M. (2012). Leadership transitions, tacit knowledge sharing and organizational generativity. Journal of knowledge management, 16(1), 45-60.
2. Csikszentmihalyi, M. (2014). New conceptions and research approaches to creativity: Implications of a systems perspective for creativity in education. In The systems model of creativity, 161-184.

3. Crisp, G. (2014). The impact of developmental education on community college persistence and vertical transfer. Community College Review, 42(2), 99-117.

4. Dyakonov, G.S. (2009). Training of engineers in a real-virtual environment of advanced training.

5. Ryzhkova, M.N. (2015). Mathematical model of the process of learning management. Bulletin of Cherepovets state University, 6 (67).

6. Ley, T. (2008). Modeling competencies for supporting work-integrated learning in knowledge work. Journal of knowledge management, 12(6), 31-47.

7. Hung, D. (2006). Engaged learning with emerging technologies.

8. Williams, P.W. (2009). The George Washington University.

9. Rahamat, R. (2011). Learners' Evaluation of an e-Learning Material. U: Proceedings of the 10th WSEAS e-Activities.

10. Berestneva, O. (2015). Students' competence assessment methods. Procedia-Social and Behavioral Sciences, 166, 296-302.

11. Ivanov, V.G. (2014). Regional experience of students' innovative and entrepreneurial competence forming. Review of European Studies, 7(1).

12. Xu, Z. (2014). A virtual reality based fire training simulator with smoke hazard assessment capacity. Advances in engineering software, 68, 1-8. 